\title{
BMJ Open The surgical burden of disease and perioperative mortality in patients admitted to hospitals in Victoria, Australia: a population-level observational study
}

To cite: Fehlberg T, Rose J, Guest GD, et al. The surgical burden of disease and perioperative mortality in patients admitted to hospitals in Victoria, Australia: a population-level observational study. BMJ Open 2019;9:e028671. doi:10.1136/ bmjopen-2018-028671

- Prepublication history and additional material for this paper are available online. To view please visit the journal (http:// dx.doi.org/10.1136/bmjopen2018-028671).

Received 3 January 2019 Revised 14 March 2019 Accepted 24 April 2019

Check for updates

(C) Author(s) (or their employer(s)) 2019. Re-use permitted under CC BY-NC. No commercial re-use. See rights and permissions. Published by BMJ.

${ }^{1}$ Department of Surgery, University Hospital Geelong, Geelong, Victoria, Australia ${ }^{2}$ Department of Plastics and Reconstructive Surgery, Johns Hopkins, Baltimore, Maryland, USA

${ }^{3}$ Royal Australasian College of Surgeons, Geelong, Victoria, Australia

${ }^{4}$ Surgery, Faculty of Health, Deakin University, Geelong, Victoria, Australia

Correspondence to Dr Trafford Fehlberg trafford.fehlberg@gmail.com

\section{ABSTRACT}

Objectives Comprehensive reporting of surgical disease burden and outcomes are vital components of resilient health systems but remain under-reported. The primary objective was to identify the Victorian surgical burden of disease necessitating treatment in a hospital or day centre, including a thorough epidemiology of surgical procedures and their respective perioperative mortality rates (POMR). Design Retrospective population-level observational study.

Setting The study was conducted in Victoria, Australia. Access to data from the Victorian Admitted Episodes Dataset was obtained using the Dr Foster Quality Investigator tool. The study included public and private facilities, including day-case facilities.

Participants From January 2014 to December 2016, all admissions with an International Statistical Classification of Diseases-10 code matched to the Global Health Estimates (GHE) disease categories were included. Primary and secondary outcome measures Admissions were assigned a primary disease category according to the 23 GHE disease categories. Surgical procedures during hospitalisations were identified using the Australian Refined Diagnosis Related Groups (AR-DRG). POMR were calculated for GHE disease categories and AR-DRG procedures.

Results A total of 4865226 admitted episodes were identified over the 3-year period. 1715862 (35.3\%) of these required a surgical procedure. The mortality rate for those undergoing a procedure was $0.42 \%$, and $1.47 \%$ for those without. The top five procedures performed per GHE category were lens procedures (162 835 cases, POMR $0.001 \%)$, caesarean delivery $(76032$ cases, POMR $0.01 \%)$, abortion with operating room procedure (65451 cases, POMR 0\%), hernia procedures (52 499 cases, POMR $0.05 \%$ ) and other knee procedures (47181 cases, POMR $0.004 \%)$.

Conclusions Conditions requiring surgery were responsible for $35.3 \%$ of the hospital admitted disease burden in Victoria, a rate higher than previously published from Sweden, New Zealand and the USA. POMR is comparable to other studies reporting individual procedures and conditions, but has been reported comprehensively across all GHE disease categories for the first time.
Strengths and limitations of this study

- This study builds on previous datasets from New Zealand, USA and Sweden, strengthening our understanding of the role surgery plays in the global burden of disease framework.

- The dataset accessed complete data from both private and public health facilities, including day procedure facilities, improving reliability.

- This study constitutes the most comprehensive report of perioperative mortality rates (POMR) to date, is among the largest cohorts of patients undergoing surgery for which POMR is reported, and reports POMR for a stable population over time.

- Limitations include uncaptured data, with an estimated $10 \%-20 \%$ of admissions not captured by using the International Statistical Classification of Diseases-10 codes for the GHE framework.

- This study accessed data through a population level administrative dataset, which are known to have limitations.

\section{INTRODUCTION}

It is well established that surgery is an essential aspect of medical care. ${ }^{12}$ Surgery is integral in both the diagnosis and management of many conditions and is needed across all age groups. ${ }^{3-5}$ It is an important part of emergency care such as bowel perforations and trauma, elective care such as uncomplicated hernia repair, and the management of chronic diseases such as peripheral vascular disease and cancer. The third Copenhagen Consensus identifies strengthening surgical services as one of the solutions to confronting the world's most important challenges, ${ }^{6}$ and surgical services play an important role towards the former Millennium Development Goals and current Sustainable Development Goals. ${ }^{7-9}$ Despite this, a significant portion of the world's population lack access to essential 
surgical services due to issues such as resource limitations, access and financial constraints. ${ }^{210-12}$

In order to advance global health, multiple organisations endorse data collection for surgical care as a first step in all countries. The World Bank and WHO recently integrated national surgical volume as a core metric of the World Development Indicators and Global Reference List of 100 Core Indicators, respectively. ${ }^{13} 14$ The Organisation for Economic Co-operation and Development independently developed guidelines to standardise surgical data elements from European countries and in 2015 the WHO unanimously passed Resolution 68/15 calling for all countries to report meaningful and reliable surgical metrics. ${ }^{1516}$

In Australia, there is emphasis on the collection and reporting of health data. Metrics relating to surgical care play an important role in measurement of the performance of the health system, quality assurance and funding distribution. Data on surgical workload, case complexity and outcomes are reported, influencing public hospital case-mix based funding. ${ }^{17} 18$ In-hospital mortality rates are reported for fractured neck of femur and emergency laparotomy for quality assurance and improvement. ${ }^{19} 20$ Emergency surgery performance indicators measure timely access to essential healthcare. ${ }^{21} 22$

Despite the importance of surgical metrics to a healthcare system, few attempts have used the WHOs data infrastructure of Global Health Estimates (GHE) to identify the global burden of disease requiring surgical intervention and no attempts have been made to calculate perioperative mortality using the GHE categorisation of disease. ${ }^{2324}$ This study's aim is to characterise the epidemiology of surgical procedures in a high-income country according to the WHOs GHE framework.

\section{METHODS}

The primary objective of this study was to identify the burden of disease requiring a surgical procedure in either a hospital or day procedure centre in Victoria, Australia. The secondary objectives were to compare incident rates of surgical procedures and calculate their perioperative mortality rates (POMR).

The data source for this study was the Victorian Admitted Episodes Dataset (VAED), a comprehensive dataset of the causes, effects and nature of illness and the use of health services in Victoria, Australia. The VAED is maintained by the Department of Health and Human Services (DHHS). It is designed to provide equitable funding and support health services planning, policy formulation and epidemiological research. A minimum set of data is provided for each admitted patient episode. All Victorian public and private hospitals are required to fulfil the reporting requirements, including rehabilitation centres, extended care facilities and day procedure centres. ${ }^{25}$ As the reporting of all admissions to the VAED is a government requirement, it is expected that the rate of missing data is negligible.

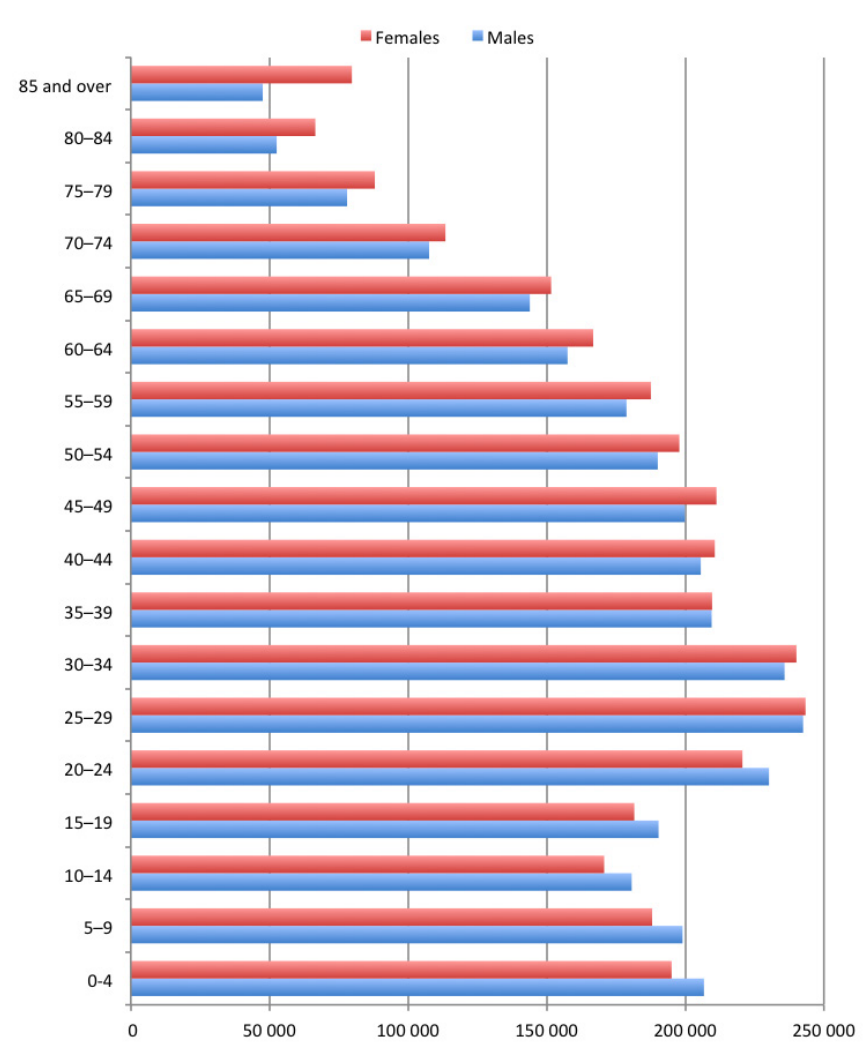

Figure 1 Victorian population by age and sex.

The Australian health system is multifaceted, with a combination of primary care facilities, acute care hospitals, day procedure facilities, rehabilitation and palliative care hospitals and outpatient services. ${ }^{26}$ There are divided responsibilities for funding involving all levels of government, private health insurers, non-government organisations and some individuals who elect to pay for services, with $68 \%$ of the total health expenditure being publicly funded in 2013-2014. In 2015, 47\% of the Australian population had some form of private health insurance. In 2013, Australia spent $9.4 \%$ of its gross domestic product on healthcare. ${ }^{27}$ Victoria is the second-most populous state in Australia and has a population density of 27 people per sq $\mathrm{km}$. Its population was 5886400 in 2014, 5996400 in 2015, and 6244200 in $2016 .^{28}$ Victoria has 320 hospitals and day procedure facilities, 151 of which are public. ${ }^{29}$ Similar to many high-income countries, the population of Australia is ageing. In 2016, $13.4 \%$ of Victoria's population was over 65 years, while $19.0 \%$ was under 15 years (figure 1$).^{30}$

Analysis of this dataset was through Dr Foster Quality Investigator (DFQI), a quality measurement tool used by the DHHS for the state of Victoria between 2014 and 2016. The database had linked data from the VAED and was designed to enable health services to monitor risk-adjusted outcomes for mortality, unplanned readmissions and length of stay. The user was able to access administrative data for admitted episodes that could be compared using factors such as diagnoses, procedure types and patient demographics. 
DFQI separates data from admitted patients into three statistical units: episodes, spells and superspells. An episode refers to a single type of care provided for a patient at a single hospital campus. The care type for an admission refers to the nature of care provided, such as acute care, rehabilitation care or palliative care. A spell refers to a continuous hospital stay at a single hospital campus, regardless of care type. A superspell is formed by concentrating contiguous spells across all health services in Victoria, both public and private. As an example, a superspell could be a patient admitted to one hospital for one episode of acute care and then discharged home, however it could also be a patient admitted to a public rural hospital for acute care who was transferred to a referral hospital for continued acute care and finally transferred to a private rehabilitation hospital for rehabilitation care. For the purposes of this paper, an admission has been defined as a superspell. ${ }^{31}$

Each admission is coded with both International Statistical Classification of Diseases 10th revision (ICD-10) codes and Australian Refined Diagnosis Related Group (AR-DRG) codes. Each admission can have multiple ICD-10 and AR-DRG codes. The AR-DRG has given each of its codes a 'medical', 'surgical' or 'other' classification. DFQI allocates a dominant diagnosis and a dominant procedure (if applicable) for each superspell. The dominant diagnosis is derived from the ICD-10 code for the principal diagnosis of the first episode. The dominant procedure is derived from the first episode that contains an AR-DRG code with a surgical classification. ${ }^{31}$ For the purpose of this paper, a surgical procedure is based on AR-DRG classification.

A search of patient admissions in the VAED between January 2014 and December 2016 was performed, separating each one into its relevant GHE disease category. To do this, a WHO data source publication ${ }^{23}$ was used which detailed the separation of ICD-10 codes into the relevant GHE disease category. The list of three digit ICD-10 codes for a given GHE disease category was then searched for using DFQI. Subsequent analysis of the group was then performed based on dominant procedure and mortality rates (figure 2). While the majority of ICD-10 codes have been assigned to a GHE disease category by the WHO, some have not been. If the dominant procedure for an admission is an ICD-10 code that is not assigned to a GHE disease category this study will not have identified that admission, resulting in uncaptured data. Based on studies of previous datasets using the ICD-10 codes for the GHE framework, it is estimated that $10 \%-20 \%$ of the total admissions may not have been captured by this study. ${ }^{3-5}$

The WHOs existing data infrastructure for comparative prioritisation of mortality, cause of death and disease burden are the GHE. The GHE comprises thousands of ICD-10 diagnosis codes apportioned into 23 disease categories which have been aggregated as: communicable, maternal, perinatal and nutritional conditions, non-communicable disease, and injuries. For each GHE disease category, those admissions that required

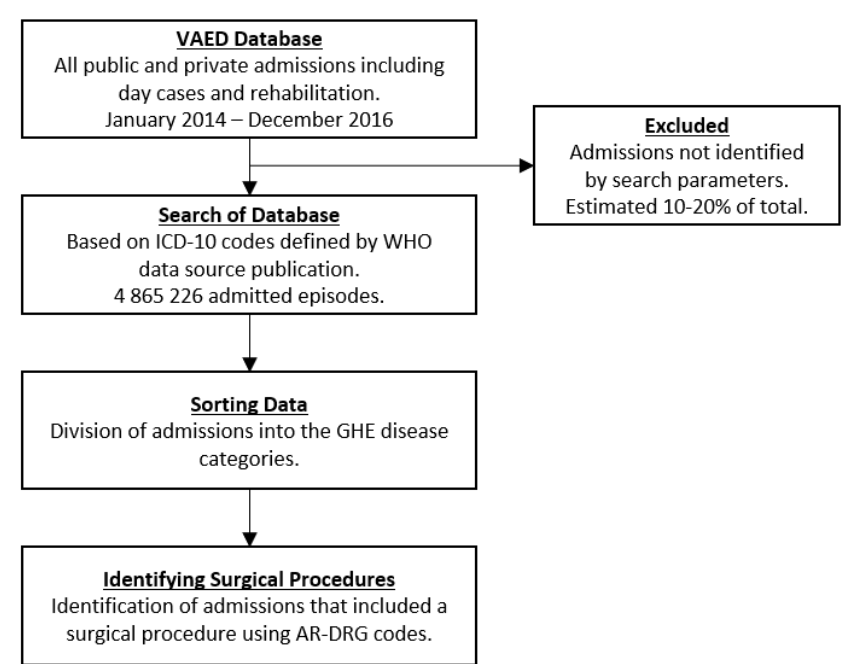

Figure 2 Flowchart of study design.

a surgical procedure were identified and the dominant procedure was recorded. Procedure rates that are quoted are the number of dominant procedures performed for any given GHE disease category rather than total number of a given procedure. This is not a typical way of reporting procedure frequency, but is useful when assessing the impact of surgical services on the global burden of disease GHE disease categories. To offset this, we have also provided information on the total number of procedures performed detailed in online supplementary appendix 1. Our method also underestimates surgical procedure frequency as only one dominant procedure can be recorded for each admission, and results should be interpreted accordingly.

In online supplementary appendix 1 , we report the 10 most frequent procedures for each GHE disease category. If the number of a given procedure was less than 100 cases, it was excluded from reporting. In 17 instances, the authors have grouped certain procedure codes together and reported as a combined number. This was performed by author consensus with the aim of improving data clarity and has been identified in online supplementary appendix 1 with a single asterisk (*). An example of this is the combination of 'open cholecystectomy' and 'laparoscopic cholecystectomy' into 'cholecystectomy'. For some GHE disease categories, more than 10 of the most frequent procedures have been reported: these extra procedures were included as they have been deemed to be of note by the authors and are identified in online supplementary appendix 1 with a double asterisk (**).

Some limitations were encountered during the data sorting process. The GHE disease categories of intentional injuries and unintentional injuries were unable to be separated, as the ICD-10 codes which define an injury as intentional or not are supplementary codes and do not map to the dominant diagnosis in DFQI. To overcome this, the two GHE disease categories were combined into a common 'injuries' and all ICD-10 codes for injuries were included, and only 22 GHE disease categories 
are reported. For the category of infection and parasitic disease subsequent analysis of the group based on individual procedures was not able to be performed before access to DFQI ended due to contractual arrangements between DHHS and DFQI.

Using excel, mortality rates were calculated by the number of events (deaths) divided by the total number of the group. This was performed for overall numbers and for each GHE disease category, subdivided by those who had a surgical procedure and those who did not, and also for each individual surgical procedure. In keeping with the WHO definition, we have defined POMR as the rate of death prior to discharge after undergoing a surgical procedure. Based off our superspell definition of an admission, our reported POMR would include a patient who died of unrelated causes in a rehabilitation hospital months after a procedure performed during an acute care episode, as long as they were transferred directly between hospitals. It would exclude day procedure patients readmitted more than 24 hours after discharge who then died from surgical complications. Unfortunately data on the length of time, the death occurred after the procedure is not available, and we are unable to report 30 day POMR. For individual procedures, we have reported PMOR for both the number of procedures performed in a particular GHE disease category, as well as the total number of procedures performed (online supplementary appendix 1). To align with previously reported similar datasets and due to the large number of separate disease states and operative categories, some of which are sex-specific, we have not separated the reporting and interpretation of the data by sex and gender.

\section{Patient and public involvement}

There was no patient or public involvement in this study.

\section{RESULTS}

During the 3-year study period, a total of 4865226 admissions were identified, with an average annual admission rate of 26839.7 per 100000 population. A total of 1715862 $(35.3 \%)$ admissions required at least one surgical procedure, resulting in an annual rate of admission requiring a surgical procedure of 9465.8 per 100000 population.

Surgical procedures were performed in all of the GHE disease categories. There was however significant variation between the categories in the rate of admission requiring a surgical procedure. The GHE disease category with the highest rate of operative frequency was sense organ diseases $(86.6 \%)$, followed by genitourinary diseases $(74.0 \%)$ and musculoskeletal diseases $(58.0 \%)$. These three categories also had the highest total number of surgical procedures; genitourinary diseases with 263637 procedures, musculoskeletal diseases with 237281 procedures and sense organ diseases with 235561 procedures. The disease category with the lowest operative frequency was mental and substance use disorders at $0.2 \%$, followed by infectious and parasitic diseases $(0.4 \%)$, neonatal conditions $(0.4 \%)$ and nutritional deficiencies $(0.4 \%)$ (figure 3).

The total number of admissions per GHE disease category ranged from 32 (sudden infant death syndrome) to 703392 (digestive diseases). The five most frequent procedures each have more admissions that the three least frequent GHE disease categories. The most frequent procedures include 162835 lens procedures, 76032 caesarean deliveries, 65451 abortion with operating room procedure, 52499 hernia procedures and 47181 other knee procedures (figure 4). The three least frequent disease categories were sudden infant death syndrome (32 admissions), diabetes mellitus (30784 admissions) and congenital anomalies (39430 admissions) (figure 3).

The mortality rate for admissions without an operative procedure was 14.7 per 1000 , while the overall POMR was 4.2 per 1000 . When this comparison is made for individual GHE disease categories, some had a much higher POMR, such as mental and substance use disorders, infectious and parasitic diseases and neonatal conditions. Other categories had a much higher mortality rate for admissions without an operative procedure, such as malignant neoplasms, respiratory diseases and cardiovascular diseases (figure 5). The 10 most frequent procedures per GHE category, which totalled $33.4 \%$ of the operative work, had a combined mortality rate of 0.1 per 1000 (figure 4). In contrast to this, the procedures with the highest mortality rates were all infrequent. When the total numbers where combined between all the GHE categories, the 10 procedures with the highest mortality rates only totalled 21192 admissions and the combined mortality rate of these was 118.3 per 1000 (online supplementary appendix 1).

For the procedures meeting inclusion criteria, the POMR is detailed in online supplementary appendix 1 for both the number of cases per GHE disease category as well as the total number of cases. For example, appendicectomy is the fourth most common procedure performed for digestive diseases, with 19623 cases and a POMR of $0.03 \%$. This comprises $96.57 \%$ of all appendicectomies performed, and the POMR remains $0.03 \%$ for total numbers. However, POMR does not always remain consistent between GHE disease categories and total numbers. Thyroid procedures are detailed for two different GHE disease categories. For endocrine, blood and immune disorders, the POMR is $0.05 \%$ (6419 cases). For malignant neoplasms, the POMR is $0.12 \%$ (1718 cases). The total number of thyroid procedures identified was 8826 , with a combined POMR of $0.08 \%$.

\section{DISCUSSION}

This study confirms previous research that surgical procedures are required across the full range of the GHE disease categories. ${ }^{3-5}$ It challenges the notion of dichotomous classification as either 'surgical' or 'medical' disease states, instead supporting the view of surgery as an integral component of a complete system of healthcare. ${ }^{5}$ 
I - Communicable, maternal, perinatal and nutritional conditions (Total admissions)
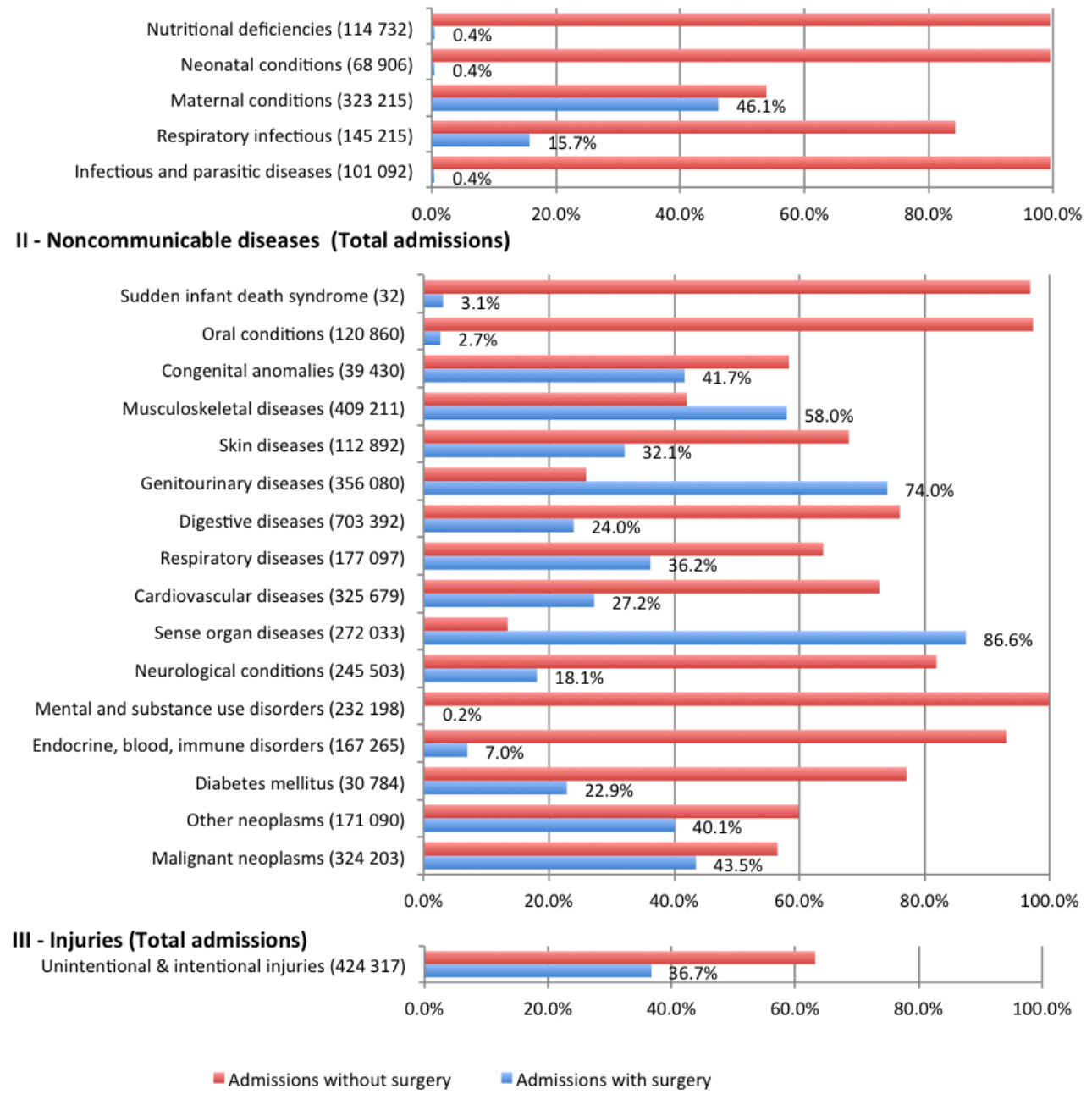

Figure 3 Global Health Estimates Disease Categories. Victorian Admitted Episodes Dataset data January 2014-December 2016.

This study also constitutes the most comprehensive report of POMR to date by including mortality rates for a large population across the full breadth of GHE disease categories.

The incident rate of surgical procedures according to the GHE framework has been calculated three times in the literature and the overall rate in Victoria (35.3\%) was higher than national cohorts in New Zealand (24.9\%), the USA, $(28.6 \%)$ and Sweden $(30.6 \%) \cdot{ }^{3-5}$ While this may represent a true higher frequency of procedures, other factors are likely to have played a significant part in this difference. For example, the Victorian dataset accessed complete data from both private and public health facilities whereas the New Zealand study by Hider et al did not capture most of the private sector. In fact, an estimated $50 \%$ of New Zealand's elective surgical volume is performed in the private sector and less than $30 \%$ of these are publicly financed. ${ }^{3}$ Our study also includes day procedure facilities which were omitted by Rose $e t a l$ in their review of the US Nationwide Inpatient Sample. In Sweden, where outpatient encounters were included, over half the surgical volume nationwide is performed in the outpatient setting. ${ }^{4}$ While we have not been able to distinguish the number of day procedures performed in this dataset, $60 \%$ of all admissions are day admissions in the Australian setting. ${ }^{29}$ This recapitulates the value of robust datasets with inpatient and outpatient procedures as well as the private sector for calculations of disease burden requiring surgery.

Additionally, definitions of surgical procedures and healthcare encounters differ between nationalised datasets. For example, our study used AR-DRG definitions of surgical codes. In the USA, over 2000 'major procedures' were defined according to the Agency for Healthcare Research and Quality. ${ }^{5}$ In New Zealand, a unique code identifies "procedures requiring general or neuroaxial anaesthesia'. ${ }^{3}$ In Sweden, the Nordic Medico-Statistical Committee classification was used as a template for thousands of major surgical procedure codes. ${ }^{4}$ The effects of these definitions are apparent in reviewing the categories of sense organ diseases and oral conditions where results differ substantially based on the definition of a 


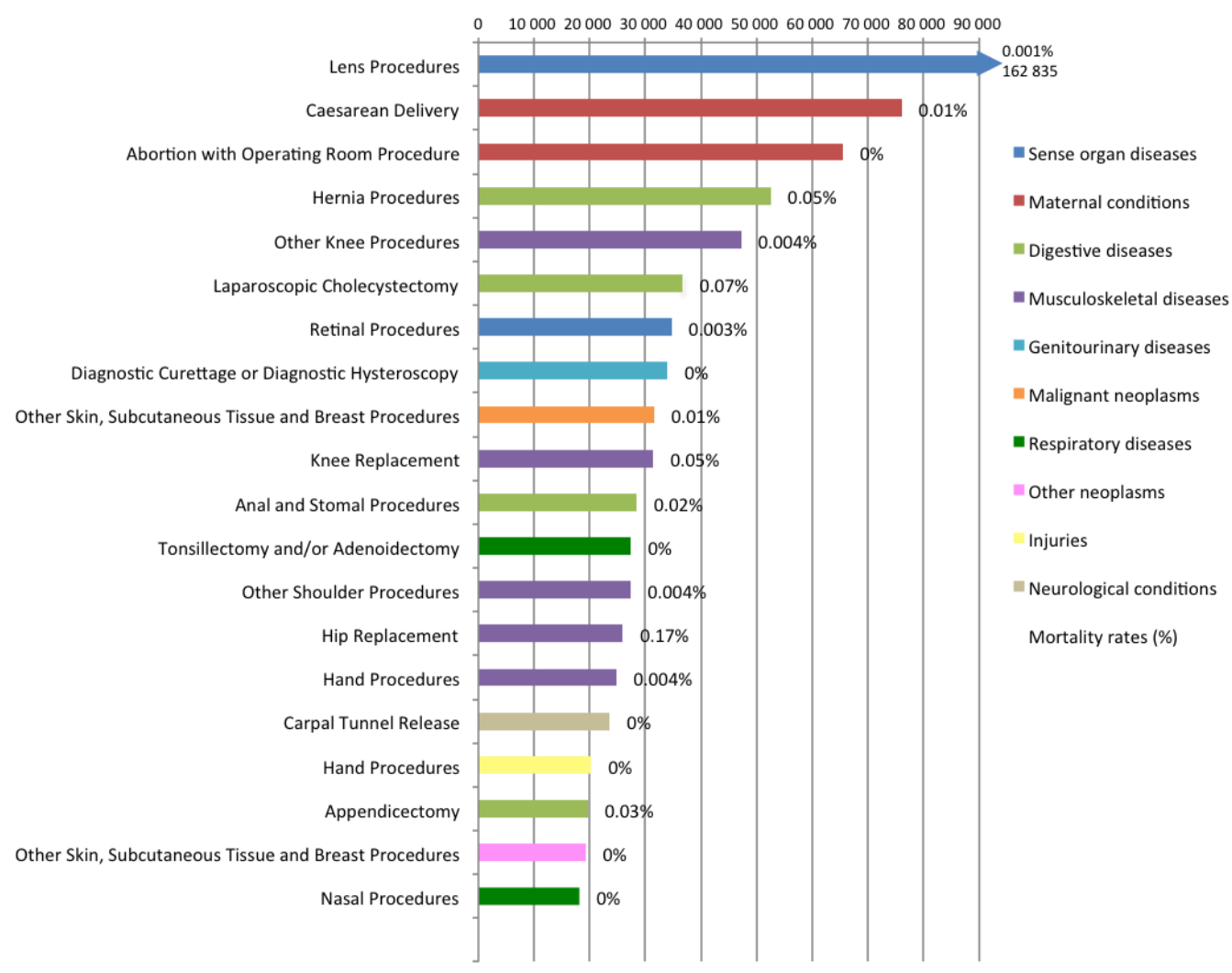

Figure 4 Twenty most frequent procedures by Global Health Estimates disease category. About $47 \%$ of total operative work.

'procedure' between New Zealand and Victorian datasets. The most frequent procedures performed in Victoria are lens procedures, which are largely not captured by the New Zealand definition of a procedure as they are usually performed under local anaesthetic. Similarly, dental extractions and restorations are very frequent procedures in the New Zealand data but they are classified as 'other' by the AR-DRG definition.

Finally, this study has defined an admission as a superspell. A patient that has been admitted to multiple different hospitals under multiple different care types during the same illness will only be reported as one admission, as long as the admissions are not separated in time by more than 24 hours. All these factors may account for differences in incident rates of surgical procedures.

This study advances the study of post-operative mortality in various ways. First, it is among the largest cohorts of patients undergoing surgery for which POMR is reported. The largest POMR cohort in the literature is a meta-analysis of 21.4 million patients by Bainbridge $e t a l .{ }^{32}$ However, this represents 87 studies with a wide variety of inclusion and exclusion criteria in diverse patient populations. This literature was updated with a subsequent meta-analysis by $\mathrm{Ng}$-Kamstra $e t$ al with a cohort of 1.0 million patients, but similarly is problematised by including 985 studies with very divergent inclusion criteria and levels of detail. ${ }^{33}$ We report inpatient POMR for 1.7 million consecutive surgical patients compared with recent reports of 46 539 patients in the European Surgical Outcomes Study
(EUSOS), ${ }^{34} 11422$ patients in the African Surgical Outcomes Study (ASOS), ${ }^{35}$ and 1.3 million in a national cohort from New Zealand. ${ }^{36}$

Second, our study has the most comprehensive scope of POMR studies to date. ${ }^{36-38}$ We report POMR for 129 discrete disease states according to the 23 categories of the WHO GHE. This level of granularity allows practitioners and policy-makers to identify areas of concern and tailer interventions to match relevant problems. For example, some GHE disease categories had a higher mortality rate for those undergoing a procedure, such as mental and substance use disorders, infectious and parasitic diseases and neonatal conditions. Other GHE categories had a much lower mortality rate for those who had an operative procedure, such as malignant neoplasms, respiratory diseases and cardiovascular diseases (figure 5). Coupling disease-specific incident rates of surgery and POMR ensures that solutions are not 'one-size-fits-all'.

Additionally, our study reports POMR for a stable population over time. A large criticism of the EUSOS and ASOS studies was that the select centres participating in the 7-day sampling period may not be representative of an entire country's surgical outcomes. ${ }^{39-45}$ Our study raises a standard of using population-level datasets to ensure representativeness of results and minimise the statistical effect of outliers. Although not possible within the scope of this study, we also advocate for analysis of trends over time, which is best achieved through large cohorts in stable populations. 


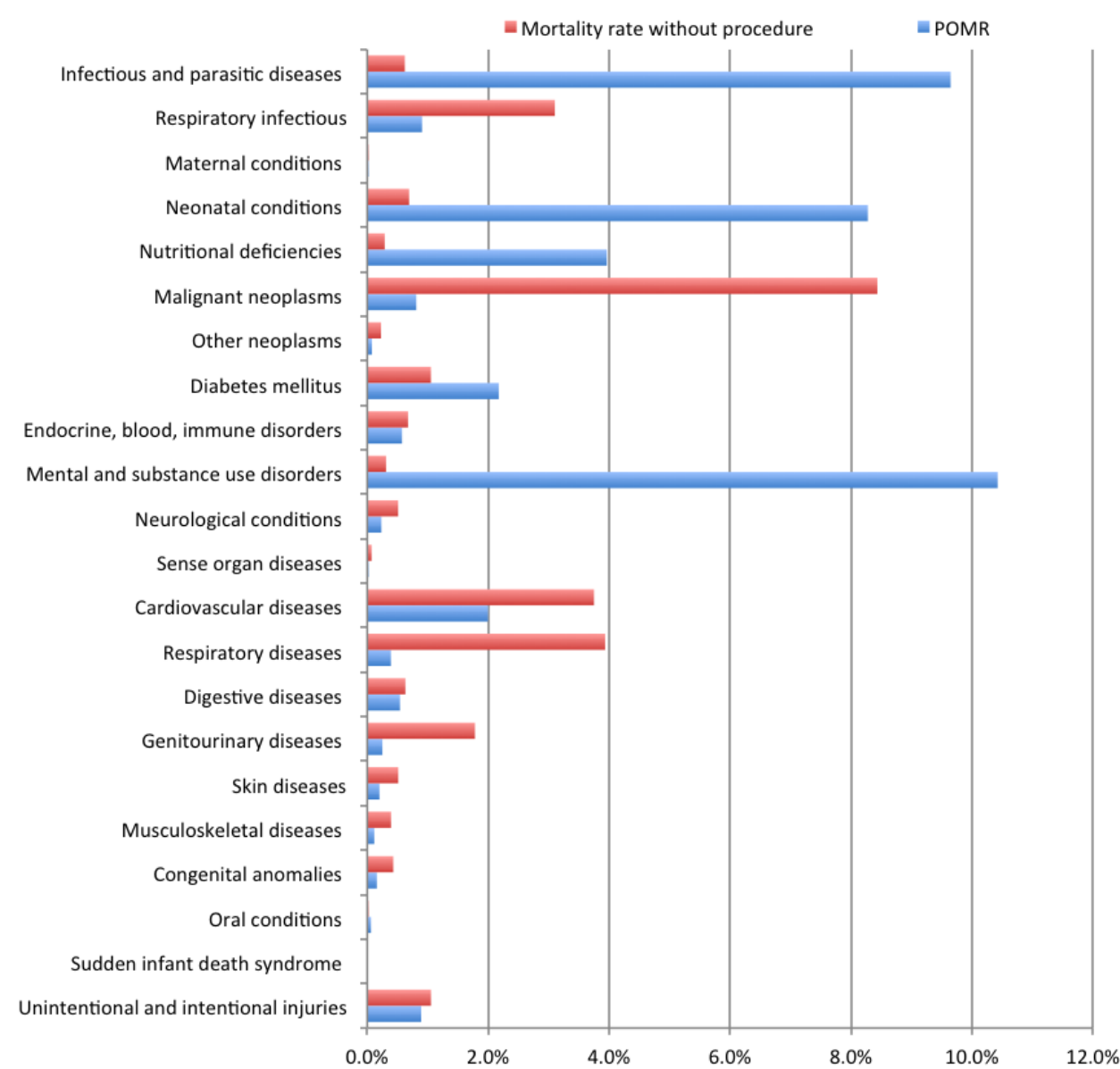

Figure 5 Mortality rates with and without procedure.

Limitations in the methodology of this study include the problem of unreported data. An estimated 10\%-20\% of admissions are not captured using the ICD-10 codes for the GHE framework, ${ }^{3-5}$ making it difficult to know how rates of operative intervention or total procedure numbers may be affected. This highlights the fact that the GHE framework was not designed to capture the surgical burden of disease. Another limitation is that the prevalence of surgical disease in the population is not captured. Instead, it captures the incidence of surgical procedures in the population admitted to hospital, including day procedure facilities. A significant proportion of the surgical burden of disease occurs in the primary care setting and may ultimately be non-operative. Third, admissions separated by time are not linked, so the same individual could have many admissions for the same illness, each of which is identified as a separate encounter. Lastly, we do not report emergency versus elective status of operations which has been shown to predict mortality risk in multiple studies. ${ }^{34-36}$

Administrative datasets carry their own limitations, such as input errors, accuracy, upcoding and downcoding. Despite these issues, administrative datasets remain an important source of population level analysis such as assessing the surgical burden of disease in the GHE framework, which would otherwise be prohibitive. ${ }^{46-48}$
Another limiting factor in the application of this dataset is the limited similar data from low-income to middle-income countries. ${ }^{49}{ }^{50}$ While data collection of this magnitude is a difficult task in a poorly resourced setting, the methodology of this study could be used to identify the surgical burden of disease in a low-income to middle-income country.

\section{CONCLUSION}

Despite these limitations, this study provides an important insight into the surgical burden of disease in patients admitted to hospital in Victoria, Australia, where over one-third of all hospitalisations required surgical intervention. It strengthens our understanding of the role of surgery in the global burden of disease framework. We have used a similar methodology to previous large population studies to confirm the role of surgery across the full spectrum of GHE disease categories. In addition, we captured complete population data as well as calculating in-hospital mortality rates overall (POMR $0.4 \%$ ) and for individual procedures. Further research is needed using a similar methodology in low-income to middle-income countries so as to inform policy makers as to planning for access to emergency and essential surgery. Identifying the surgical component of disease managed in a primary 
healthcare setting, using the GHE categorisation, would also be beneficial in assessing the true prevalence of surgical disease.

Acknowledgements The authors would like to thank C Brown for his contribution and assistance with the data. This study has not been previously presented at a conference or preregistered with an independent institutional registry.

Contributors All authors meet ICMJE requirements for authorship. DW devised the project, DW and TF developed the main conceptual ideas and design; JR and GDG contributed to the design of the project. TF acquired the data. TF, DW and JR performed the analysis and interpretation of data. TF drafted the manuscript and $\mathrm{JR}, \mathrm{GDG}$ and DW revised it critically. Final approval was provided by TF, JR, GDG and DW.

Funding The authors have not declared a specific grant for this research from any funding agency in the public, commercial or not-for-profit sectors.

Competing interests None declared.

Patient consent for publication Not required.

Ethics approval Ethical approval was granted by the Doctor Foster Clinical Focus Group within the DHHS for Victoria. This focus group was comprised of a committee of senior clinicians from different health services, delegated by DHHS to provide oversignt of the use of DFQI, including providing independent ethical and publication approvat of related research projects.

Provenance and peer review Not commissioned; externally peer reviewed.

Data sharing statement A de-identified summary of the data is available for use in Appendix 1, no additional data is available.

Open access This is an open access article distributed in accordance with the Creative Commons Attribution Non Commercial (CC BY-NC 4.0) license, which permits others to distribute, remix, adapt, build upon this work non-commercially, and license their derivative works on different terms, provided the original work is properly cited, appropriate credit is given, any changes made indicated, and the use is non-commercial. See: http://creativecommons.org/licenses/by-nc/4.0/.

\section{REFERENCES}

1. Debas HT, Donkor P, Gawande A, et al. Disease Control Priorities, Third Edition : Volume 1. Essential Surgery. Washington, DC: World Bank, 2015.

2. Meara JG, Leather AJM, Hagander L, et al. Global Surgery 2030: evidence and solutions for achieving health, welfare, and economic development. The Lancet 2015;386:569-624.

3. Hider P, Wilson L, Rose J, et al. The role of facility-based surgical services in addressing the national burden of disease in New Zealand: An index of surgical incidence based on country-specific disease prevalence. Surgery 2015;158:44-54.

4. Omling E, Jarnheimer A, Rose J, et al. Population-based incidence rate of inpatient and outpatient surgical procedures in a high-income country. Br J Surg 2018;105:86-95.

5. Rose J, Chang DC, Weiser TG, et al. The role of surgery in global health: analysis of United States inpatient procedure frequency by condition using the Global Burden of Disease 2010 framework. PLoS One 2014;9:e89693.

6. Third Copenhagen Consensus Outcomes Document. Copenhagen, Denmark: Copenhagen Consensus, 2012.

7. Rosa W. Transforming Our World: The 2030 Agenda for Sustainable Development. A New Era in Global Health. New York, NY: Springer Publishing Company, 2017.

8. The PLoS Medicine Editors. A Crucial Role for Surgery in Reaching the UN Millennium Development Goals. PLoS Medicine 2008;5:e182.

9. United Nations. The Millennium Development Goals Report, 2015.

10. Alkire BC, Raykar NP, Shrime MG, et al. Global access to surgical care: a modelling study. Lancet Glob Health 2015;3:e316-e323.

11. Ouma PO, Maina J, Thuranira PN, et al. Access to emergency hospital care provided by the public sector in sub-Saharan Africa in 2015: a geocoded inventory and spatial analysis. Lancet Glob Health 2018;6:e342-e350.

12. Rose J, Weiser TG, Hider P, et al. Estimated need for surgery worldwide based on prevalence of diseases: a modelling strategy for the WHO Global Health Estimate. Lancet Glob Health 2015;3(Suppl 2):S13-S20.

13. Global Reference List of 100 Core Health Indicators. 2015. http:// www.who.int/healthinfo/indicators/2015/en/ (accessed 1 Jun 2018).
14. World Development Indicators 2016. 2016. https://data.worldbank. org/products/wdi (accessed 1 Jun 2018).

15. Lafortune G, Balestat G, Durand A. OECD Health Division. Comparing activities and performance of the hospital sector in Europe: how many surgical procedures performed as inpatient and day cases? https://www.oecd.org/health/Comparing-activities-andperformance-of-the-hospital-sector-in-Europe Inpatient-and-daycases-surgical-procedures.pdf (accessed 11 Aug 2018).

16. Resolution A 68/15: Strengthening emergency and essential surgical care and anaesthesia as a component of universal health coverage. 2015. http://apps.who.int/gb/ebwha/pdf_files/WHA68/A68_R15-en. pdf?ua $=1$ (accessed 13 Aug 2018)

17. Broughton KJ, Aldridge O, Pradhan S, et al. The Perth Emergency Laparotomy Audit: The Perth Emergency Laparotomy Audit. ANZ Journal of Surgery 2017;87:893-7.

18. Chia PH, Gualano L, Seevanayagam S, et al. Outcomes following fractured neck of femurin an Australian metropolitan teaching hospital. Bone Joint Res 2013;2:162-8.

19. Marshall T, Suthersan M, Chan MK, et al. Mortality and morbidity of neck of femur fractures: A comparison between Orange and peripheral centres: Mortality and Morbidity of Fractures. Australian Journal of Rural Health 2016;24:253-7.

20. Stevens CL, Brown C, Watters DAK. Measuring Outcomes of Clinical Care: Victorian Emergency Laparotomy Audit Using Quality Investigator. World J Surg 2018;42:1981-7.

21. Parasyn AD, Truskett PG, Bennett M, et al. Acute-care surgical service: a change in culture. ANZ J Surg 2009;79:12-18.

22. Stupart DA, Watters DA, Guest GD, et al. Dedicated emergency theatres improve service delivery and surgeons' job satisfaction: Emergency general surgery theatre sessions. ANZ Journal of Surgery 2013;83:549-53

23. WHO methods and data sources for global burden of disease estimates 2000 - 2015. Geneva: World Health Organisation, Department of Information, Evidence and Research, 2017.

24. Abbott TEF, Fowler AJ, Dobbs TD, et al. Frequency of surgical treatment and related hospital procedures in the UK: a national ecological study using hospital episode statistics. $\mathrm{Br} J$ Anaesth 2017;119:249-57.

25. Victorian Admitted Episodes Dataset (VAED) manual, 27th Edition, Section 1 - Introduction. 2017. https://www2.health.vic.gov.au/ hospitals-and-health-services/data-reporting/health-data-standardssystems/data-collections/vaed (accessed 1 Jun 2018).

26. Hall J. Australian Health Care--The Challenge of Reform in a Fragmented System. N Engl J Med 2015;373:493-7.

27. Australia's Health 2016. Canberra: Australian Institute of Health and Welfare, 2016.

28. Australian Bureau of Statistics. Australian Demographic Statistics 2017. 2018. http://www.abs.gov.au/AUSSTATS/abs@. nsf/second+level+view?ReadForm\&prodno $=3101.0 \&$ viewtitle $=$ Australian\%20Demographic\%20Statistics Dec\%202017 Latest 21/ 06/2018\&\&tabname $=$ Past $\% 20$ Future $\% 20$ lssues\&prodno $=3101.0 \&$ issue $=\mathrm{Dec} \% 202017$ \&num $=\&$ view $=$

29. Australia's hospitals $2015-16$ at a glance. 2017. https://www.aihw. gov.au/reports/hospitals/ahs-2015-16-at-a-glance/contents/table-ofcontents (accessed 9 Oct 2018).

30. ABS data.. Population by Age and Sex, Regions of Australia 2016. 2017. http://www.abs.gov.au/ausstats/abs@.nsf/Latestproducts/ 3235.0Main\%20Features252016?opendocument\&tabname= Summary\&prodno $=3235.0 \&$ issue $=2016 \&$ num $=\&$ view (accessed 8 Jun 2018).

31. Goldsworthy C. Quality Investigator Victoria Business Rules September 2015, 2015.

32. Bainbridge $\mathrm{D}$, Martin J, Arango M, et al. Perioperative and anaesthetic-related mortality in developed and developing countries: a systematic review and meta-analysis. Lancet 2012;380:1075-81.

33. Ng-Kamstra JS, Arya S, Greenberg SLM, et al. Perioperative mortality rates in low-income and middle-income countries: a systematic review and meta-analysis. BMJ Glob Health 2018;3:e000810.

34. Pearse RM, Moreno RP, Bauer P, et al. Mortality after surgery in Europe: a 7 day cohort study. The Lancet 2012;380:1059-65.

35. Biccard BM, Madiba TE, Kluyts HL, et al. Perioperative patient outcomes in the African Surgical Outcomes Study: a 7-day prospective observational cohort study. Lancet 2018;391:1589-98.

36. Ariyaratnam R, Palmqvist CL, Hider P, et al. Toward a standard approach to measurement and reporting of perioperative mortality rate as a global indicator for surgery. Surgery 2015;158:17-26.

37. Davies JF, Lenglet A, van Wijhe M, et al. Perioperative mortality: Analysis of 3 years of operative data across 7 general surgical projects of Médecins Sans Frontières in Democratic Republic 
of Congo, Central African Republic, and South Sudan. Surgery 2016;159:1269-78.

38. Uribe-Leitz T, Jaramillo J, Maurer L, et al. Variability in mortality following caesarean delivery, appendectomy, and groin hernia repair in low-income and middle-income countries: a systematic review and analysis of published data. Lancet Glob Health 2016;4:e165-e174.

39. Brodner G, Van Aken H. Mortality after surgery in Europe. The Lancet 2013;381:370.

40. Doherty S, Conroy RM, Dunne H. Mortality after surgery in Ireland. The Lancet 2063;2013:382.

41. Franek $E$, Osinska $B$, Czech $M$, et al. Mortality after surgery in Europe. The Lancet 2013;381:369-70.

42. Mikstacki A. Mortality after surgery in Europe. The Lancet 2013;381:369.

43. Pearse R, Moreno RP, Bauer P, et al. Mortality after surgery in Europe - Authors' reply. The Lancet 2013;381:370-1.

44. Pupelis G, Vanags I. Mortality after surgery in Europe. The Lancet 2013;381:369.
45. van Schalkwyk JM, Campbell D. Mortality after surgery in Europe. The Lancet 2013;381:370.

46. Gavrielov-Yusim N, Friger M. Use of administrative medical databases in population-based research. J Epidemiol Community Health 2014;68:283-7.

47. Pongpirul K, Robinson C. Hospital manipulations in the DRG system: a systematic scoping review. 2013;7:10.

48. Schneeweiss S, Avorn J. A review of uses of health care utilization databases for epidemiologic research on therapeutics. J Clin Epidemiol 2005;58:323-37.

49. Anderson GA, Ilcisin L, Abesiga L, et al. Surgical volume and postoperative mortality rate at a referral hospital in Western Uganda: Measuring the Lancet Commission on Global Surgery indicators in low-resource settings. Surgery 2017;161:1710-9.

50. Riviello R, Scott JW. Closing the data gaps for surgical care delivery in LMICs. Lancet Glob Health 2016;4:e138-e139. 